\title{
Enhancing Creativity in Interaction Design: Alternative Design Brief
}

\author{
Corina Sas and Alan Dix \\ Computing Department, Lancaster University, InfoLab21, South Drive, \\ Lancaster, LA1 4WA, UK \\ \{corina,dixa\}@comp.lancs.ac.uk
}

\begin{abstract}
This paper offers a critique of the design brief as it is currently used in teaching interaction design and proposes an alternative way of developing it. Such a design brief requires the exploration of alternative application domains for an already developed technology. The paper presents a case study where such a novel type of design brief has been offered to the students taking part in a collaborative design project and discusses how it supported divergent thinking and creativity as well as helped enhancing the learning objectives.
\end{abstract}

\section{Introduction}

The importance of design brief on the entire design cycle cannot be overstated. This paper advocates that alternative ways of writing a design brief can support creative thinking which in turn contributes to better design outcomes. The proposed design brief encourages the exploration of new application domains for an already developed technology. Through addressing some of the limitations of the traditional design brief, it contributes to the improve ment of teaching and learning interaction design. The presented case study is focused on a collaborative design project module delivered to Master students enrolled in a Human-Computer Interaction (HCI) program.

The paper starts by outlining the challenges of teaching interaction design. This is followed by an introduction of the concept of design brief together with the main limitations of its current format that has been traditionally used in interaction design education. From these limitations, an alternative way of preparing a design brief is proposed. The case study offers a detailed description of the proposed design brief together with an in depth reflection on its benefits among which the exploration of design space is particularly highlighted. 


\section{Teaching Interaction Design}

Interaction design is primarily a creative process which despite the efforts made to explore it, raises significant questions, i.e., the strategies involved in complex and illdefined problems, the trade-off between conflicting design constraints, or the nature of creative insight and how it can be replicated and supported. It is this limited understanding that leads to difficulties in teaching interaction design. The challenges of teaching design can be seen throughout the entire design process, starting from problem specification, continuing with the relevant feedback that the students need to receive, and not at least relating to the assessment of design-related activities and their outcomes [1].

\subsection{Challenges}

This section highlights three main challenges related to teaching/learning interaction design which focus on the tension between ambiguity and structure within the problem specification, on the temporality of the relevant feedback that the students need to receive, e.g. continual/continuous feedback; and on the objectivity of design assessment, both in terms of design outcomes and design process.

Problem specification in the context of interaction design brings into attention aspects like ambiguity and structure. While educators try to provide just enough details to leave room for the exploration of the design space, students prefer a more articulated and structured problem definition [2]. This tension is generated by students' limited ability to handle less structured tasks. However, the skill to formulate problems precedes and is at least as important as the one of finding solutions. Unfortunately, in today's higher education, the emphasis is almost entirely placed on problem solving skills, while significantly less efforts have been made to ensure the acquisition of problem formulation skills [3]. From our experience of teaching interaction design, even graduate students are often less prepared for this challenge; it is a skill to be learned.

Setting the problem is needed not only during the initial stage of the design process since its iterative nature requires continuous reformulation and restructuring of the problem all along the design cycle $[4,5]$. Ultimately, problem specification challenges the educator to decide what the right level of detail is.

Students' learning can hardly progress without efficient feedback. However, providing relevant feedback during the design process is a challenging endeavor which relates both to its timely quality [4] and feedback content [6]. The former refers not only to the continual feedback throughout the design process, but also to the continuous feedback during the critical phases of the design cycle. In order to provide efficient and timely design guidance, the educators need to be involved in the critical decision points along the entire design process. This is usually not a trivial task since in order to progress efficiently students need to show initiative to organize their work within and mostly outside the mentoring sessions [6].

The difficulty of objectively assessing the quality of design outcomes represents another challenge in teaching design which impacts also on the quality and content of the educator's feedback. This is due mainly to the difficulty of evaluating any 
design outcomes as being right or wrong since the design decisions that have to be made will always leave unexplored a multitude of options against which the current outcome can never be compared [6]. Of course, several prototypes can be evaluated through user trails and compared against each other but this is not enough to claim that a particular design is the best that can be produced. The evaluation of the design process (as opposed to design outcomes) is even more problematic, if one wants to go beyond the design stages and wishes to explore the considered design options and the rationale for deciding which one has been followed. The breadth and depth of the design space exploration usually goes against the limited resources of the design project. In addition, there is no current methodology that can account for the insightful conversion of a set of design constraints into affordances.

The importance that we choose to pay to the design brief is due to the fact that the design brief ultimately dictates how the design process is going to unfold. Given their significance, it is surprising how little work has focused on how design briefs should be prepared. The few attempts in this direction usually come from industrial arena and are introduced in the following section.

\subsection{Professional vs. Academic Design Brief}

Design briefs represent concise descriptions of a required design task. The briefs offer information on the design problem and its context, and require engagement in creative problem solving activities with the purpose of providing solutions for the design problem. The key element of the design briefs developed for educational purposes, e.g. interaction design programmes, is the provision of just enough structure which should enable a strong focus on the design process and students' reflection on it, rather than on the design outcomes.

On the other hand, the design briefs developed in business \& industrial sector (professional design briefs) focus mainly on the expected outcomes of the design, while supporting the comprehensive understanding of the problem that needs to be solved [7]. Therefore, the design brief should consist of a thorough presentation of the problem, together with the expected outcomes of design, and it should answer questions such as:

- Why are we doing this project?

- Why are we doing it now?

- What specific outcomes are to be expected?

- Who are we designing for?

- Who are the key stakeholders in this project?

- What are the phases of this design project?

- How much time must be devoted to each phase?

- Who will approve the final design solution?

- What criteria will be used for this approval?

- How will the design solution be implemented?

- How will the results be measured?

The above questions could provide also a template for writing design briefs in the academic arena. In fact, some of the details offered by the answers to these questions are usually included in the narrative of a design brief. For example, below 
are outlined the core ideas of the briefs from the CHI Student Design Competition for the last three years:

- Design a service to promote the use of public transit (CHI 2007).

- Design a service for personal monitoring of diet, exercise and health for individuals (CHI 2006).

- Design a tool, application or service for elder companionship (CHI 2005).

These examples reflect the standard format of the design brief written for teaching/learning purposes. This consists mainly of the design problem that outlines the motivation for finding an adequate solution to address it [8, 9, 10]. Within this format, the students are given a real life topic with its own affordances and constraints and are asked to design a suitable system to address the proposed topic. While widely accepted, this type of design brief has several limitations, as identified during our experience of mentoring collaborative design projects of Master students enrolled in a HCI program.

a) Generic problems like the ones presented above are challenging since the design space could be extremely large. While students feel comfortable in exploring it, they usually lack the skills of narrowing down the problem in order to confine it to a limited range of constraints and affordances. In other words, a lot of time and effort is spent in redefining and narrowing the problem at the expense at the time needed to find alternative solutions for the identified problem. Consequently, once the problem is defined in more concrete terms, the students prefer to go ahead to refine the first acceptable option with little interest in further exploring alternative solutions.

b) Following from the above limitation, such a design brief is always at risk of providing not enough structure. In a generic problem there appears to be a challenge in providing the right level of detail.

c) The inefficient exploration of the design space can also act as a barrier for student creativity, divergent thinking and problem solving, since more time is spent in narrowing down the generic problem rather than exploring creative solutions to the design problems.

d) The time constraints that impact on any design project dictate that for a ten weekproject after exploring the design space, narrowing the problem and working on the conceptual design, little time is left for prototyping the solution. Therefore, the students do not progress much in refining their ideas, and usually stop at low fidelity prototypes, e.g. paper prototypes.

e) Another limitation relates to the relevant aspect of feedback that the students need from their educators both in terms of its quality and timely characteristic.

f) The assessment of the quality of design outcomes is a major challenge in teaching design [5]. The problems relate to the rather large design space which is usually limitedly and unsystematically explored within student projects [6]. One way to address these limitations, besides a rigorous management of the time schedule, is through exploring alternative types of design briefs. 


\section{3 Proposed Design Brief}

We propose a design brief which requires students to find new ways of using an already developed technology. In other words, the focus is placed on finding $a$ problem for a given solution rather than finding solutions for a given problems. Although this approach is currently used in industry, its role in teaching and learning design has not been explored.

The proposed design brief may sound just like technology- or business-driven design, which is normally denigrated within the HCI community. Perhaps because of this, the more positive uses of a technology focus are often missed. In a poorly conceived business-driven design which attempts to identify new markets for a particular technology, the technology on offer is foisted on to a user group without regard to its appropriateness to their needs. It is certainly true that technology should always match the user needs. However, it is not necessary that this is achieved by starting from needs and progressing to appropriate technology. In our case, we are using the technology to seek for potential design settings where such a match between needs and technology exists. It is simply the case that the order is reversed.

The following section offers a description of a case study where the new type of design brief has been offered to students taking a collaborative design project module.

\section{Case Study}

\subsection{Study Module}

The Collaborative Design Project (CDP) module is part of a Master by Research (MRes) program which represents a collaborative initiative between the departments of Psychology and Computing at Lancaster University. The overall program focuses on the development of research skills in designing and evaluating interactive systems. The program runs for a small group of students, e.g. less than a dozen, who are usually highly motivated, sometimes with couple of years of industrial experience, and with educational backgrounds in Psychology, Computer Science, Information Technology and sometimes Arts. This is a fortunate mixture which resembles some of the multidisciplinary nature of real design teams.

Within this program, the CDP focuses on collaborative design of an interactive system. It is a compulsory module which runs for 10 weeks and worth $10 \%$ of the degree. This CDP module is structured to support a constructivist approach to learning [7]. While mainly a collaborative project-based learning, e.g. students usually work in groups of three or four with mixed educational backgrounds, the CDP module presents also some elements of problem-based learning.

By the end of the module, students produce low fidelity prototypes or simulations rather than working prototypes. The assessment procedure involves three tasks: two individual reports in which students reflect on the design outcome and the collaborative nature of the design process respectively. In addition they have to 
prepare a website and a poster that needs to be explained to a panel of experts during group presentation.

\subsection{Proposed Design Brief}

We experimented with the new type of design brief in the 2005-2006 academic year. Below is an excerpt of the design brief:

This design project will focus on the technology developed in the "Pin and Play" project running in Computing Department (http://ubicomp.lancs.ac.uk/pin\&play/overview.html). The latter focuses on developing a general architecture for constructing physical interfaces that can be ad hoc composed and adapted by their users.

Your task is to explore alternative, innovative ways of exploiting this technology, through a set of applications, others than those already developed within this project. For this, you are expected to generate a large pool of possible applications. After their evaluation, you will select couple of them and designed them in greater details. The mains constraints are defined by the technology already developed.

You will be working in two groups, each group involving participants with psychology and computer science background to enrich the pool of group resources. Each team will focus on a different theme, targeting two different dimensions of human activity. One group will search for applications aimed towards supporting work, e.g. probably in office environment but not only, while the other team will try to design applications supporting play, e.g. in indoor public spaces but not only. The teams will collaborate, in terms of evaluating each other's design outcomes.

The rationale for choosing the Pin and Play technology relates to its versatility, since this technology gradually became a growing toolkit for exploring tangible interactive surfaces. A brief description of this technology is outlined below.

Pin and Play is characterized by an augmented surface, which provides data connectivity and tangible interactive artifacts that can be added to or removed from the surface. When an artifact is added, it becomes connected, acquires a digital representation and can be manipulated while it is on the surface, providing thus a link between the digital and the physical worlds. The surface provides the connectivity and the physical support to enable this.

\section{Discussion}

The design process entailed by the proposed brief consists of three phases. This section describes in details each phase together with its outcomes and benefits.

The Phase I involved intense collaboration between students and the researchers in the department involved in the development of Pin and Play technology. The opportunity to enter into a dialog with these researchers ensured students' access to first hand knowledge and experience. They learned to ask the right questions in order to develop a thorough understanding of the system. 
Apart from engaging in a dialog with the researchers developing Pin and Play, the students also had the opportunity to play around with the system itself. This experiential component $[9,11,12]$ added significant value by enriching students' knowledge of the system functionalities, constraints, affordances as well as look and feel. In addition, the tangible dimension of Pin and Play itself added value to this experience.

Through this process of inquiry and experiential learning, the students identified a large set of constraints related to system functionality. The thorough understanding of the Pin and Play system allowed students to identify a set of the system constraints which is outlined below.

- Maximum number of components that can be used simultaneously

- Component operations and delays

- Grouping components

- Mapping components to actions

- Transitioning on knobs/sliders

- Size of the fabric

- Short circuits

- Spatial awareness

- Prongs and safety

- Power requirements

- Data transfer

- Miscellaneous

The identification of constraints took place alongside with a literature review activity. For this, each group put together a list of academic papers to ensure that the most important areas to focus on are well represented, and to avoid any duplication of efforts that otherwise may occur. Subsequently, each student reviewed three papers and entered the reviews on the group website, thus making his/her work available to the other members of the group. During the literature review, students experienced a first recognition of the user needs as captured within the literature review.

Once the constraints were identified and the state-of-the-art explored, the students started to engage in the development of a large pool of ideas. Thus, by the end of week five, i.e., half way within the project cycle, each group produced around 20 conceptual designs as alternative ways in which Pin and Play technology could be used. This was an important step within the design cycle that lasted almost three weeks. The ideas were developed as conceptual designs, each with several low details paper prototypes. During this phase, the students succeeded in developing ideas that indicate a broad exploration of the design space. This could only be possible through intense divergent thinking (Fig. 1).

Indeed, brainstorming sessions were particularly encouraged at this stage, which represented the most exploratory stage of the design process. This initial phase was the least constrained one (and arguably the most creative), since the creative process being has focused on the ill-defined problem without any attempt of assessing the ideas. 


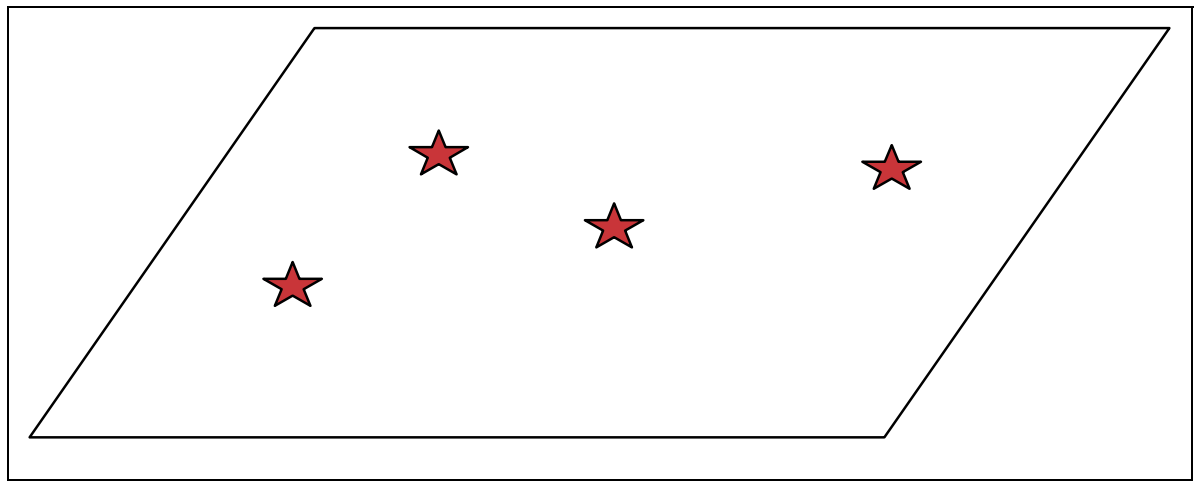

Fig. 1. The Phase I of the design cycle involved a broad exploration of the design space. The stars represent the explored ideas in different areas of the design space

The explored dimensions associated with the conceptual designs are outlined below. This presentation starts with the conceptual designs and prototypes developed by the group focused on identifying application domains to support work activities.

- Supporting collaborative tasks like information retrieval, participatory design, or ontology building.

- Supporting fluid interactions between digital and physical artifacts in applications like tangible desk or customizable mouse.

- Supporting exploration of the physicality for multiple selection tasks like order interface of assembly line-style sushi bar, or dynamic timeline wall.

- Mapping for navigating at ease around a map to be used as an educational tool, or for crime investigation.

- Supporting scheduling like Outlook/iCal assistant, and collaborative notice board.

- Supporting security like secure door entry, or tangible safe.

- Exploring graphic/sound aid in the form of photo filter tool, or garage production studio.

Below are the conceptual designs and prototypes developed by the group focused on identifying application domains to support play, fun, learning and entertainment.

- Simulations of real world systems like dress selector, 3D outfit, board game interface or garden design.

- Educational support like electronic circuit game, pin doctor.

- Language development like language education board, spatial sound board.

- Domestic control tools.

- Interactive games like hangman game, pin potato head or customizable pin and playball game.

- Multiplayer games like pin \& fight, multiplayer etch a sketch.

- Memory based games like "PinPlay says" or safe cracker game.

To summarize, the main dimensions within the design space that have been covered are the following:

- use of Pin and Play for both individual and collaborative tasks; 
- use of the fabric of Pin and Play both in the traditional two dimensions as well as in three dimensions;

- use of Pin and Play both in stationary and dynamic/mobile way; and

- use the spatial awareness which Pin and Play empowers its users with.

In this way, the main outcome of the Phase I consists of identifying system constraints, the main dimensions of the design space and the large pool of ideas exploring the design space in the forms of seven conceptual designs with three prototypes each as alternative applications for Pin and Play. The major benefit of this Phase consists of encouraging students' divergent thinking in exploring broadly the design space.

Phase II can be viewed as a breaking point between the other two phases since it requires students to stop and assess their work before moving forward with an indepth exploration of the design space. Thus, once the conceptual designs/prototypes were developed the Phase II involved their assessment.

The main outcomes of this phase consisted of a list of criteria developed for assessing the conceptual designs and their associated prototypes. For this, each group has prepared a leaflet where one page was dedicated to each conceptual design or prototype. The criteria outlined below were proposed by the students and refined with input from the course tutor.

- Functionality.

- Information and clarity of the concept.

- Integration between the conceptual design and the user goals.

- Successful exploration of the haptic and tangible aspects of the system.

- Originality and novelty.

- Overall satisfaction.

The assessment was carried out in week 5 by a team of academic staff and researchers in the department and has led to the identification of a winner conceptual design (Fig. 2).

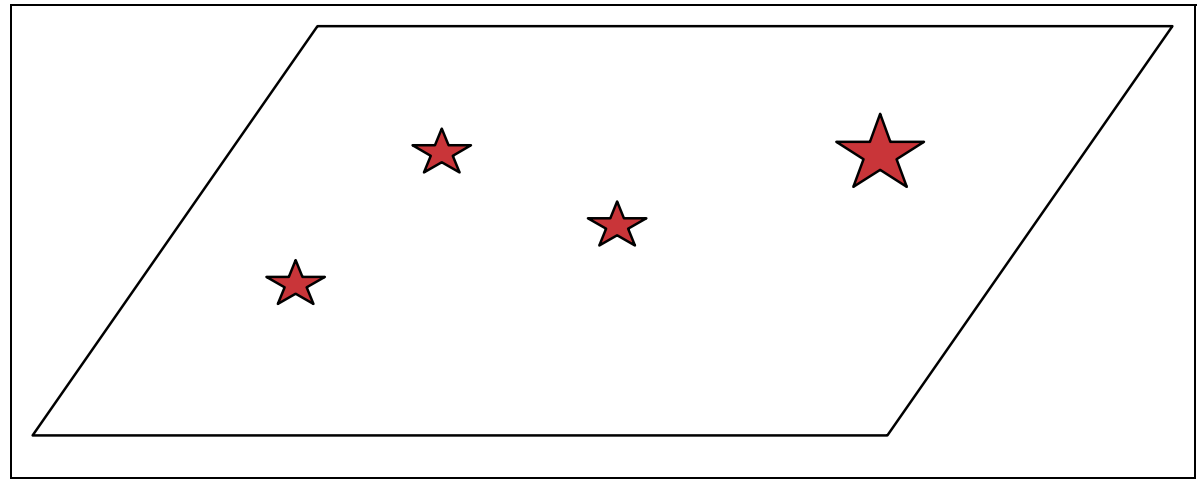

Fig. 2. The Phase II of the design cycle enables the identification of the conceptual design that successfully meets the assessment criteria. The larger star represents the conceptual design selected among the competing ones. 
The benefits of the developed criteria consist of making the selection phase more objective which in the end also helped the assessment/marking process for this module. Through engaging students in formulating the assessment criteria they became involved in critical reflection and in a movement from the mere "I like it" to an understanding of the dimensions of the problem space. This is in itself a significant and often neglected aspect of design.

Phase III consisted in refining the selected conceptual design and its associated prototype. The main outcomes of this phase are encapsulated in students' final prototypes.

One group produced a video prototype which outlined a complete game scenario involving a doll, pins and other artifacts as physical prototypes. In the PinDoctor application which is a tangible medical game, the child plays the role of a doctor whose task is to diagnose and treat the patient: a doll made of the fabric of Pin and Play. The interactive component embedded in the game allows the doctor to play with various types of pins for performing medical tasks, e.g. injecting drugs, sampling blood. The pins inserted into the patient fabric could be consequently used to perform two functions. One is to provide diagnostic information such as temperature or heart rate (Fig. 3). The other is to provide treatment through administering medicine. The pins change their state as the game progresses and the feedback is provided by sounds from the doll and through the visual information from GUI. Particular emphasis is placed on the sequence of events, e.g. diagnosis of symptom and treatment, and the spatial location of action, e.g. if a drip is administered in the correct location.

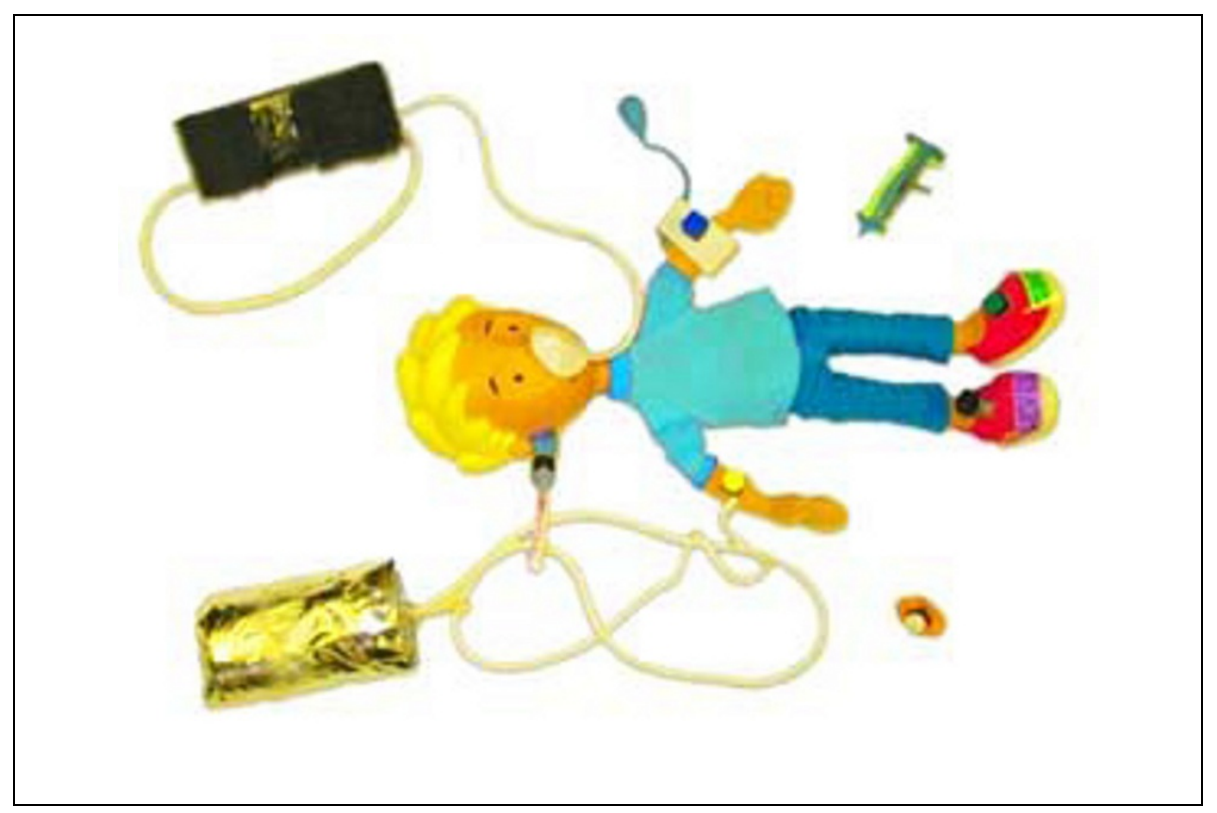

Fig. 3. PinDoctor physical prototype 
The other group produced a working prototype which required understanding the code behind the Pin and Play and the ability to integrate new code in the existing system. Pin \& Search is a tangible collaborative tool for information retrieval aiming to offer a standard interface outputting search results from a database people wish to search from. Initially a number of keywords are entered into the system, printed on cards and associated with pins. Consequently, when a tagged pin is entered into the fabric of a collaborative board, the search starts. It can be changed as pins are added on or removed from the fabric. The search results are displayed on a screen located alongside the collaborative board, and they can be further manipulated through the use of navigation controls.

This depth in the design cycle has not been previously reached by the former cohorts of students exposed to the traditional design brief (who usually stopped at the level of paper prototyping). This outlines the benefit of this phase which enabled a thorough exploration of the design space. Such exploration has been possible due to the stronger starting point, i.e., an already developed technology rather than an abstract idea (Fig. 4).

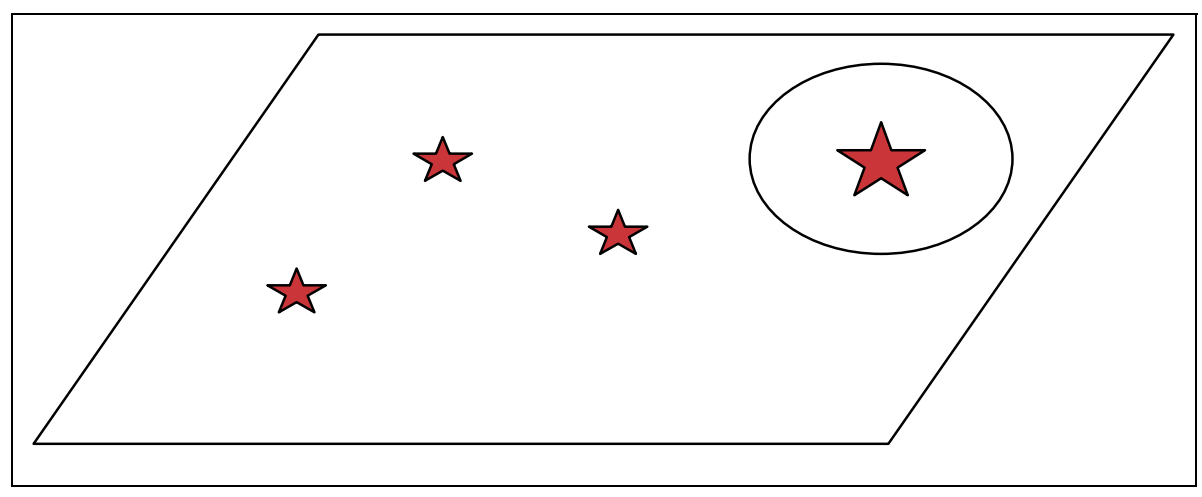

Fig. 4. The Phase III of the design cycle enables an in-depth exploration of the design space in the area around the conceptual design selected in Phase II.

It is indeed, the exploration of design space that represents the main advantage of this type of design project. The traditional design brief can be summarized as follows:

Generic Problem $\rightarrow$

Specific problem $\rightarrow$

Brief exploration of solutions $\rightarrow$

Selection of technology $\rightarrow$

Prototyping a solution.

The proposed design brief can be represented like:

Existing Technology $\rightarrow$

Understanding the technology $\rightarrow$

Exploration of how it can be used $\rightarrow$

Choosing "a problem" $\rightarrow$

Prototyping a solution. 
Whereas the greater amount of time in the first case is spent on specifying the problem, the second case allows the greater amount of time to be spent on the exploration of alternative ways of using the technology and prototyping the chosen solution. This exploration ensures a broad coverage of the design space, whereas the work on prototyping the selected solution ensures an in-depth exploration of the design space.

To summarize, the main outcomes of this case study that enriched students learning experience consists of:

- The acquisition of a set of transferable skills for inquiring the developers about the existing technology.

- The thorough knowledge about this technology which led to a comprehensive set of constraints.

- The development of skills required to elaborate a list of criteria that supports the objective evaluation of the conceptual designs, as well as the transparent assessment of the entire module.

- The development of knowledge and skills required to produce a large number of conceptual designs, e.g. seven for each group with 2-3 sketched prototypes for each concept.

- The development of knowledge and skills required to produce a refined version of the prototype for the selected conceptual design, e.g. a video and a working prototype respectively.

- Enhanced creativity throughout the design process.

\section{Conclusions}

Each of the design challenges of teaching interaction design that were identified in first section are revisited below and suggestions are made into how the proposed design brief can address them.

The replacement of a generic problem with an already developed technology offers a different starting point in exploring the design space. Rather than having an abstract idea, students are faced with a tangible working system. The concreteness of an existing system is an advantage that cannot be surpassed by a design brief that is purely imagined.

The time budget can be better used for generating and assessing multiple solutions rather than stopping after the first one has been found.

This type of brief, particularly when the technology proposed has been developed in the department offers additional benefits. It allows students' access to an ongoing departmental project which enables the development of transferable skills, such as scientific inquiry, building on research expertise, and communication skills. In this way, the students benefit from more mentoring, both from the module tutor and from the research team involved in developing the existing technology. Thus, the amount of feedback that they receive along the design cycle is substantial. In other words, this type of brief enables students' access to a community of practice [11, 12], namely the one in the department. 
The proposed design brief supports transparency and objectivity in assessing students' work, through facilitating students' involvement in developing assessment criteria.

The proposed brief can be seen as part of a larger family of approaches to design, those involving the ready to use technology from the very beginning of the design process. This allows for a strong experiential learning component [13] to be part of the initial exploratory phase which in turn increases the quality of the design process. However, while in this paper the ready to use technologies represents a starting point leading to new incarnations for different application domains, tasks and user needs, i.e., Pin and Play; in other situations it can be more a means to an end such as mediators facilitating fast prototyping, i.e., the phidgets developed by Saul Greenberg at University of Calgary (http://grouplab.cpsc.ucalgary.ca/phidgets).

Beside the benefits outlined above, the proposed brief has also limitations that need to be mentioned. Indeed, the proposed type of design brief is particularly relevant for innovative technologies such Pin and Play. For an existing technology such as the use of the web, there will be the danger that the students will be blinkered by previous experience of its applications. In such cases, more radical means, for example BadIdeas [14] may be needed in order to encourage divergent thinking during the exploration phase. A limitation of the evaluation process taking place in Phase II consists of its lack of objective criteria. Future work can address this limitation by including expert evaluation of the conceptual designs.

Not at least, the quality and the number of the developed conceptual designs suggest the advantage of this type of design brief both for supporting divergent thinking and for allowing a better exploration of design space both in breadth and in depth.

\section{Acknowledgements}

Our thanks to the MRes students 2005-2006 for their enthusiasm and hard work when faced with this new type of design brief, and to Nicolas Villar for generously sharing his time and knowledge about Pin and Play.

\section{References}

[1] C. Sas, Learning Approaches for Teaching Interaction Design, in: Proceedings of HCIEd.2006-1 Inventivity, Limerick, Ireland, 23-24 March, 2006.

[2] D. Schön, Educating the reflective practitioner (Jossey Bass, London, 1987).

[3] Frederiksen, N. Implications of Cognitive Theory for Instruction in Problem Solving. Review of Educational Research, 54(3), 363-407 (1984).

[4] C. Kehoe. Supporting Critical Design Dialog, Ph.D. Dissertation, Georgia Institute of Technology, 2001 (unpublished).

[5] D.A. Wroblewski, The construction of human-computer interfaces considered as a craft ,in: Taking software design seriously, edited by J. Karat (Academic Press, Cambridge, 1991), pp. 1-19. 
[6] G. Strong, J.B. Gasen, T. Hewett, D. Hix, J. Morris, M.J. Muller, and D.G. Novick, New Directions in HCI Education, Research, and Practice, (Washington, DC: NSF/ARPA, 1994).

[7] P.L. Phillips, Creating the Perfect Design Brief: How to manage design for strategic advantage (Allworth Press, New York, 2004).

[8] B. Hartfield, T. Winograd, and J. Bennett, Learning HCI design: Mentoring project groups in a course on human-computer interaction, SIGCSE Bulletin, 24(1), 246-251 (1992).

[9] S. Howard, User interface design and HCI: identifying the training needs of practitioners, SIGCHI Bulletin, 27(3), 17-22 (1995).

[10] D. Benyon, P. Turner, and S. Turner, Designing Interactive Systems (Addison-Wesley, London, 2005).

[11] J. Lave and E. Wenger, Situated Learning: Legitimate Peripheral Participation (Cambridge University Press, Cambridge, 1991).

[12] E, Wenger, Communities of Practice: Learning, Meaning, and Identity (Cambridge University Press, Cambridge, 1998).

[13] J. Piaget, The Psychology of Intelligence (Routledge, New York, 1950).

[14] A. Dix, T. Ormerod, M. Twidale, C. Sas, P. Gomes da Silva, and L. McKnight, Why bad ideas are a good idea. Proceedings of HCIEd.2006-1 Inventivity, Limerick, Ireland, 23-24 March, 2006. 